\title{
Design of Fuzzy Algorithm Control Based on MFC and Control of Tank Liquid Level System
}

\author{
Da Huang*, Shucai Huang, Wei Zhao and Wenhuan Cao \\ Air Force Engineering University, Xi’an 710051, China \\ ${ }^{*}$ Corresponding author
}

\begin{abstract}
PID control algorithm has the advantages of precision and stability, but it is difficult to meet the requirements of complex control, and the improved PID algorithm is in urgent need of development. Fuzzy PID algorithm combined with the advantages of fuzzy and PID, has the characteristics of fast and stable, its application is more extensive. Controller is a kind of encapsulation of the data, method and interface technology, this paper use VC6.0 design controller of fuzzy PID algorithm, in the "zutaiwang" running environment of double let water tank liquid level control, the experiment shows that the fuzzy PID control algorithm is compared with PID control, faster to achieve stability, and both are no steady-state error. The control algorithm control is easy to use and is an effective packaging for the algorithm.
\end{abstract} tank

Keywords-PID; fuzzy-PID; ActiveX controls; double let water

\section{INTRODUCTION}

Nowadays, control systems are more complex, and simple control methods cannot meet the changing system requirements [1]. Due to the advantages of stability and precision, PID has a dominant position in the control field. With the complexity of the control system, the PID improvement method has been developed. Fuzzy PID is one of the improved algorithms of PID. It has very practical performance and is widely used. In the paper, the application of fuzzy PID control in automatic pouring system is studied[2]. Wang xinli et al. used PID to control the artificial leg, and its control has good gait following [3]. Liu shuai studies the control method of internal formation track keeping, and its method is mainly based on PID, including traditional PID and nonlinear PID [4]. Wu kui used fuzzy PID to adjust the power of the computer monitoring system of hydropower station, and its has good control effect and robustness [5]. The simulation results show that the fuzzy PID control has good anti-interference [6]. Fuzzy PID control is applied to the improvement of hydraulic system of stepping beam, which effectively solves the problem of stepping beam in work [7]. Zou ling and other design fuzzy self-tuning PID controller, and use it in reheating steam temperature control [8]; Wang haiqing modeled and simulated the fuzzy self-tuning PID temperature control system and improved the performance of the temperature control system [9].

Control technology, a kind of encapsulation of the data, methods, and interface technology, is a powerful way to develop software [10], the paper uses VC6.0 to design control of fuzzy PID. And the control was carried out through the liquid level control experiment of double-volume tank, and was verified

\section{CONTROL ALGORITHM THEORY}

\section{A. PID Control Algorithm}

The model of PID is expressed as follows [11]:

$$
u(t)=K_{p}\left[e(t)+\frac{1}{T_{i}} \int e(t) d t+T_{d} \frac{d e(t)}{d t}\right]
$$

Where $u(t)$ is the output of the controller, $e(t)$ is the deviation signal, $\mathrm{Kp}$ is the proportional coefficient of the controller, $\mathrm{Ti}$ is the integral time constant of the controller, and $\mathrm{Td}$ is the differential time constant of the controller.

PID control model is made up of proportion, integral and differential. The proportion of controlled directly, that is, whether the system reaches stability depends on how fast proportional control part [5]. Proper Kp should be selected to achieve stable and fast effect. The integral uses the accumulation of small deviation to control the system to achieve the error free effect; Differential is controlled by the variation of deviation and plays a major role in system antiinterference.

The PID control algorithm for computer operation is discretized, and its expression is as follows:

$$
u(k)=K_{p}\left[e(k)+\frac{T_{s}}{T_{i}} \sum_{i=0}^{k} e(i)+T_{d} \frac{e(k)-e(k-1)}{T s}\right]
$$

Where $u(k)$ represents the output of $k$ sampling period, and $\mathrm{e}(\mathrm{k})$ represents the deviation of $\mathrm{k}$ sampling period, and Ts is the sampling period.

Suppose $K_{I}=K_{p} \frac{T s}{T_{i}} ; K_{D}=K_{p} \frac{T_{d}}{T s}$

There are:

$$
u(k)=K_{p} e(k)+K_{I} \sum_{i=0}^{k} e(i)+K_{D}[\mathrm{e}(\mathrm{k})-\mathrm{e}(\mathrm{k}-1)]
$$




\section{B. Fuzzy PID Control Algorithm}

Fuzzy PID algorithm is applied to self-tuning of PID parameters to achieve better control [12]. It includes the fuzzy algorithm advantages of fast and intelligent, and the stability and accuracy of PID, which is an improvement of traditional PID. Its basic principle is to accurate input value, and then according to the fuzzy rules, determine output, the output of the identified at this time is a fuzzy value, and finally the blur to determine the accurate output values (PID parameters), the principle block diagram is shown in Fig.1.

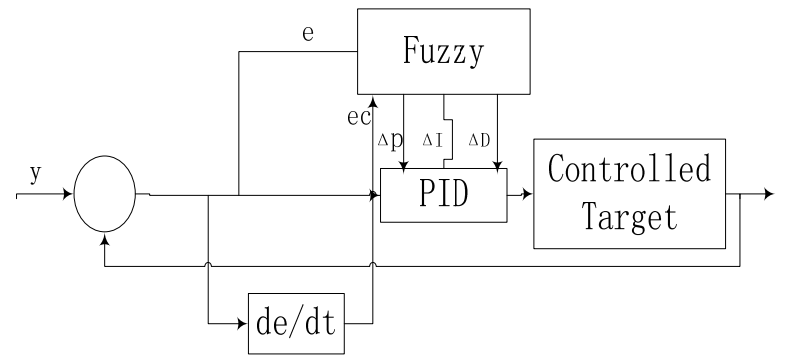

FIGURE I. SCHEMATIC DIAGRAM OF FUZZY PID CONTROLLER

\section{IMPLEMENTATION OF ACTIVEX CONTROL}

Using tool of MFC in vc6.0 to write ActiveX control, the basic framework includes $C^{*} \mathrm{App}, \mathrm{C}^{*} \mathrm{Ctrl}, \mathrm{C}^{*}$ Prop Page. Where $\mathrm{C}^{*} \mathrm{App}$ is the application class; $\mathrm{C}^{*} \mathrm{Ctrl}$ is the control class, that is, the main content of the design control is implemented in this section. The $\mathrm{C}^{*}$ Prop Page class is derived from the Cole Property Page and is used to write the properties Page program. Basic framework, there are two functions, one is STDAPI Dll Register Server (void) functions, its role is to control the information written to the registry, the other is a STDAPI Dll Unregister Server (void) functions, its role uninstall registration information. In addition, $\mathrm{D}^{* *}$ and _ $\mathrm{D}^{* *}$ Events in the framework, which represent interfaces, are used to complete the communication between the container and the control. The implementation flow chart of ActiveX control is shown in Fig.2.

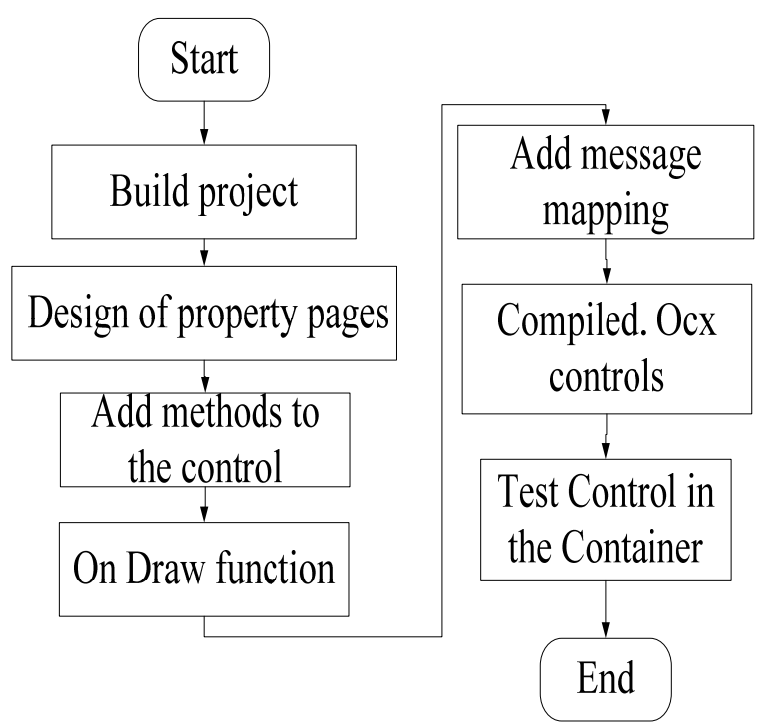

FIGURE II. FLOW CHART OF CONTROL DESIGN
- Use MFC ActiveX Contrl Wizard to create a new project called PID. Open the vc6.0 -> file -> project-> MFC ActiveX Control Wizard, enter the PID in the project name and click "Ok", and leave the contents in the dialog box that pops up by default, and click "finish"-> “Ok";

- Add attributes to the control. Click "view" in the toolbar -- >" Class Wizard"; The MFC Class Wizard dialog box will pop up. Click "Automation” - > “Add Property", the external name in the pop-up dialog input variables, corresponding algorithm to add attributes to variables associated with the container, so in the class view DPID will appear under the defined properties, and can appear under PID control c m_kp corresponding member variables and properties.

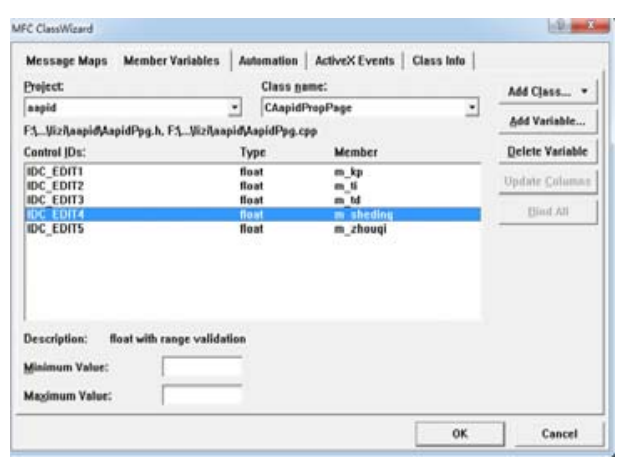

FIGURE III. DIALOG OF MFC CLASS WIZARD

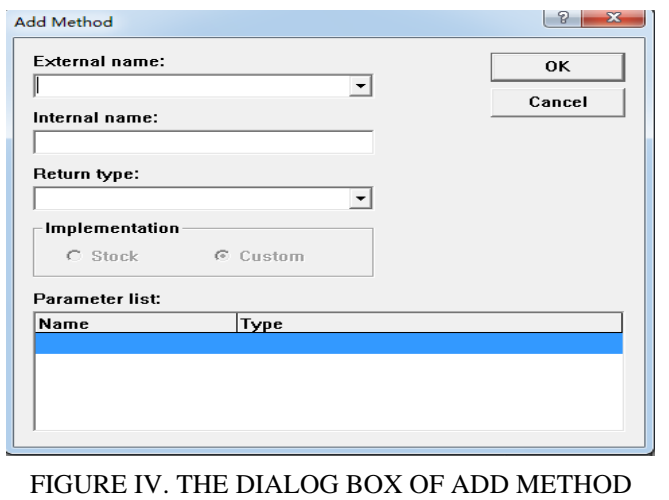

- Design of property pages. When you add properties to a control, you need to add the edit box corresponding to the attribute variable in the property page so that you can change the value of the control property in the property page. Open Resource View - > Dialog - > IDD_ PROPPAGE _ PID, add Member Variables to the corresponding edit box and is associated with properties, right click on the edit box, select the Class Wizard, open the Member Variables, in the name of the Class to choose CPID Prop Page Class, the Dialog box shown in Fig.3. Dialog box to add member variables respectively, and the value of the note is that in the add member variable dialog with Optional property name column, the column is the edit box in the properties page associated with attribute set, in this column to join associated variables such as KP. 
- Add methods to the control. Control is a function of the Method, it can be regarded as the main function of the function, through the MFC Class Wizard - > Automation - > Add Method, will appear the dialog box shown in Fig.4, the function name part of the input pid, the return type is void, pid( ) will appear in the CPIDCtrl Class, Add the pid algorithm in the corresponding position.

- $\quad$ On Draw function. The interface design of the control is completed here.

- $\quad$ Add message mapping. Add WM_CREATE message handler, WM_TIMER two t breath I response function, the purpose is to make the control interface updates, by adding the WM_CREATE message handler in the CPID Ctrl class message processing, in the generated response function On the Create () to add a Timer, add WM_TIMER message processing, added to draw in the On the Timer function. Add pid () in On Create(), call pid method, implement control algorithm;

- Compiled. Ocx controls, after the success of the compilation, will see in the debug window "Registering ActiveX Control... The ocx control is registered and can be used directly.

- Test Control in the Container, can in VC6.0 software shipped Container Test Control, through the Tool - > Activex Control Test Container, open the Test Container, in the Test Container toolbar to open the edit - > insert new Control, the dialog box shown in Fig.5 appears, find the writing your own controls;

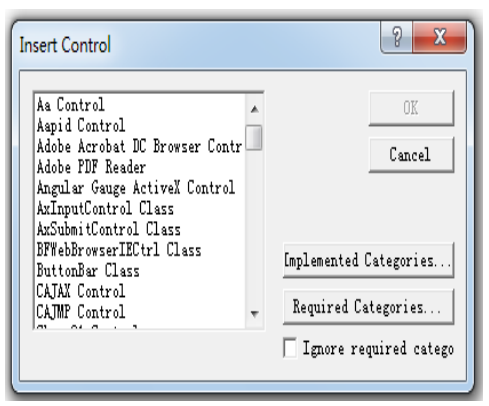

FIGURE V. INSERTS THE CONTROL DIALOG BOX

\section{EXPERIMENT AND ANALYSIS}

\section{A. Experimental Environment}

The experimental device contains four water tanks, each of which has a liquid level sensor. The device can provide water tank level control experiment, and its schematic diagram is shown in Fig.6.

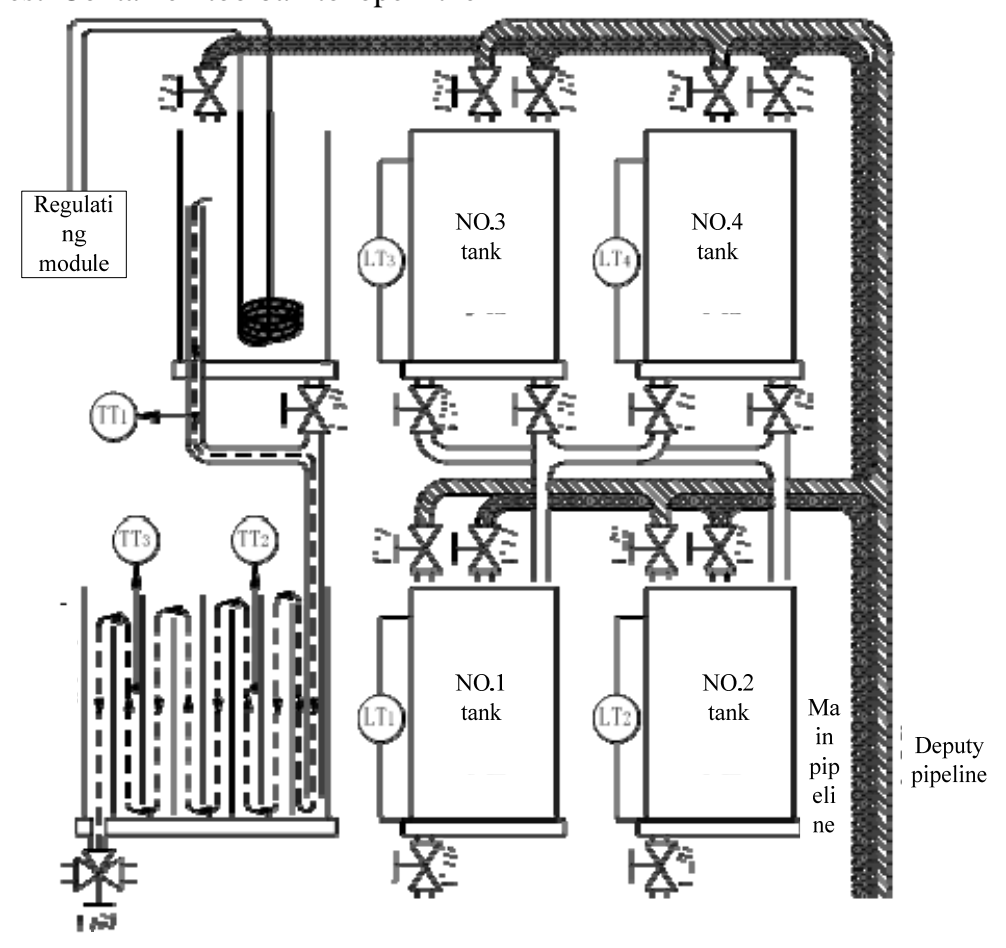

FIGURE VI. SCHEMATIC DIAGRAM OF EXPERIMENTAL DEVICE

The test procedure for the liquid level of the dual capacity tank is as follows:
- Select the executing agency. As can be seen from the figure, the system has two executing agencies 
("U1"and"U2") to choose from, and one of them can be selected as the control quantity;

- Select the double objects, as shown in the above, the system includes four water tank, water tank 1 and 2 water tanks, water tanks, 3, 4 water tanks, can choose a set of $(1,2$, or 2,4$)$ double let water tank as the system under test;

- Compose the control loop, open the corresponding valve according to the selected control quantity and water tank, and compose the control loop;

- Select the working point, so that the system is in a normal working state and the tank level reaches a certain stable point;

- Control step change, on the basis of step (4), change the set value, and observe the change of liquid level of the controlled water tank.

The controls written in this design run the tests in such a system. The liquid level control of dual capacity tank has the characteristics of hysteresis and non-linearity, and the control test experiment is representative in such a system.

"zutaiwang" is a kind of computer based configuration software, with stable, real-time performance characteristics, and it is easy to use, and is recognized by people. In this design, an experimental equipment is used to form a liquid level control system of a double-volume tank, and the ActiveX control is called in the "configuration king" to control the system. The flow diagram of the control step designed by the king of configuration is shown in Fig.7.

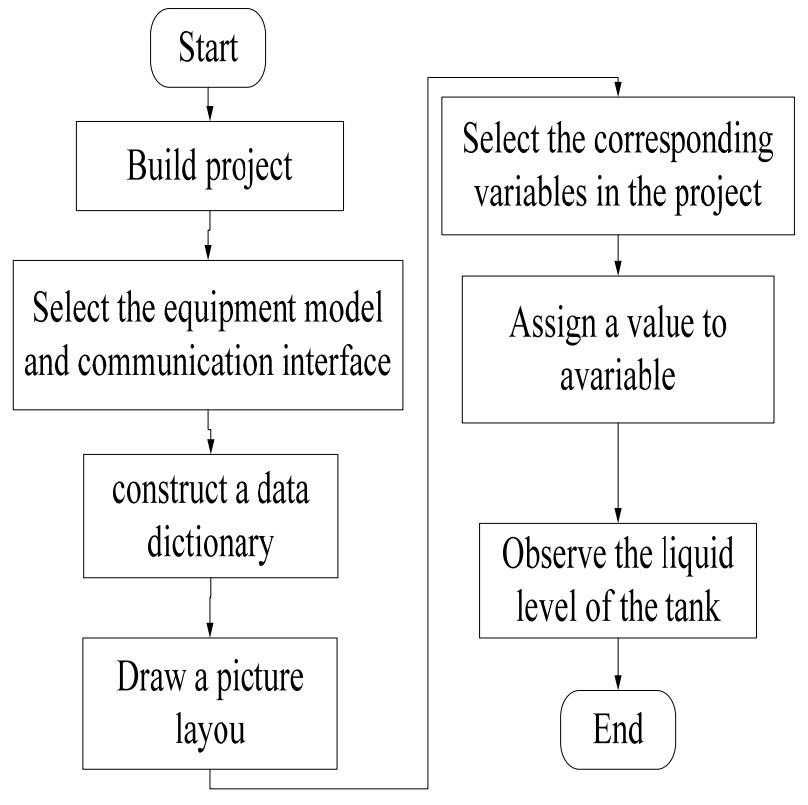

FIGURE VII. CONTROL FLOW CHART

- $\quad$ Open software and create a new project;

- Select the equipment model and communication interface, and the purpose is to associate the king with experimental instruments to ensure the communication between them;

- To construct a data dictionary, which is to define the variable (IO or memory type) used in the project, such as setting, input, output, and so on. The project will automatically allocate memory for it. To allow data to be transmitted.

- Draw a picture layout, create a painting surface, find the control option, click; Select the control you wrote, double-click, insert the control. Rearrange the text to change P,I, and D parameters.

- Select the corresponding variables in the project, such as text KP, which can be connected to the $\mathrm{m} \_\mathrm{kp}$ in the control. Select and connect the dependent variables for each property of the control. There are three Settings (see figure 4.3) for the relation direction of the attribute variables, and the correlation direction must be set, otherwise the control will not run properly.

\section{B. Results and Analysis}

After the control test of three controls, the significance of writing in the control field is further proved. According to the test procedure of the liquid level test of the double-volume tank, the effect of PID control and fuzzy PID control was analyzed and compared, and the liquid level history curve of water tank 1 was obtained as shown in Fig 8.

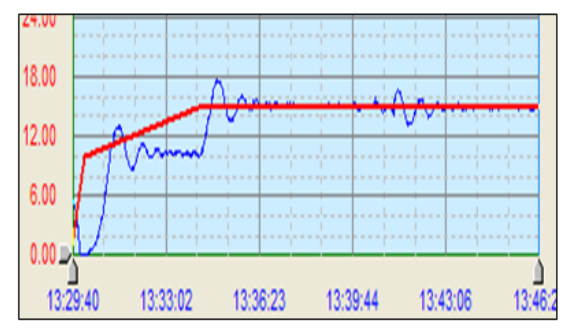

FIGURE VIII. THE LIQUID LEVEL CURVE OF PID

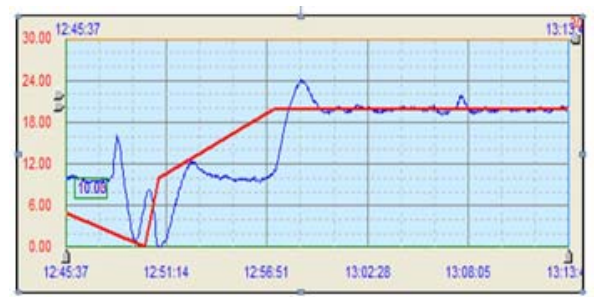

FIGURE IX. THE LIQUID LEVEL CURVE OF FUZZY PID TANK 1

Fig.8 shows that in 13:29:50 moment,when Set the liquid level to be $10 \mathrm{~cm}$, after 3.5 minutes the level reaches a steady state level curve, at 13:34:05 time, change the set point to 15 $\mathrm{cm}$, after 3 minutes level curve is stable, at 13:41:00 time, increase the water flow as an interference, after 2 minutes level of stability.

From Fig.9 can be seen, in 12:51:10 moments, when Set the liquid level to be $10 \mathrm{~cm}$, after 2.6 minutes reach a steady state level curve, in the moment 12:56:53, change the set point to 20 $\mathrm{cm}, 2.8$ minutes after stable level curve, at 13:07:30 time, 
increase the water flow as an interference, over 50 seconds level of stability.

Look from the experiment results of the analysis, the traditional PID control can achieve stability, no error control effect, but poor anti-jamming performance compared with the fuzzy PID control, and in the process of experiment, the artificial parameter setting was complicated. The fuzzy PID control is fast, without error and strong anti-interference. The experimental results are in accordance with the theoretical reasoning.

\section{SUMMARY}

The paper analysis of control algorithm of PID and fuzzy PID, and uses VC6.0 control wraps, describes in detail the development processes and controls in configuration software used in the container. The experiment of control shows that the control can realize the function of PID and fuzzy PID algorithm, and it is convenient to use and has certain engineering significance. The experimental results show that fuzzy PID is better than PID, which is consistent with theoretical analysis. Control design has the following significance: (1) the convenient control algorithm of calls, this design in software "zutaiwang" pilot run, double let water tank level control experiment was carried out, completed the control objectives, of course, can also be in other application calls this control controls; (2) a better finished the application of the control algorithm, when implement some algorithm in configuration or similar container, no need to write a lot of algorithm in configuration or similar container program, just call. Ocx control can reach the goal; (3) the control can be compatible with a variety of containers, that is to say, only a machine language (such as c language) can be used to write an algorithm program, and a variety of containers (programs) can be used to run the algorithm.

\section{REFERENCES}

[1] W. Shunhuang, before shudi, intelligent control system and application [M], mechanical industry press, 2015.

[2] S Wenyou, C An, H Yueming, Design of Embedded Vision System on High-power LED Automatic Glue-Pouring Devices[J];Instrument Technique and Sensor;2012-10.

[3] W xinli. Modeling and PID control of mechanical prosthesis [J]. Journal of dalian jiaotong university, 2013,38(4):125-129.

[4] L shuai. Research on the control method of the internal formation track of the team [D]. National university of defense science and technology.

[5] W kui. Fuzzy PID power regulation of computer monitoring system of hydropower station [J]. Journal of chongqing university,2004,27(5):1727.

[6] Z chun. Design and research of PID parameter fuzzy self-tuning controller [J]. Mechanical and electrical engineering,2006,23(9):19-21.

[7] M Yang. Transformation of hydraulic system for stepping beam based on fuzzy PID control [J]. Manufacturing automation, 2012,35 (12) :8-11.

[8] Z ling. Application of fuzzy self-tuning PID controller in reheating steam temperature control [J]. Control system,2005,21(12):25-26.

[9] W haiqing, ji changying et al. Modeling and simulation of fuzzy selftuning PID temperature control system [J]. Computer engineering,2012,38(7):233-239.

[10] L yu-min. Development of advanced control algorithm in configuration software [D]. Daqing petroleum institute.2005.

[11] W shunhuang, shudi. Intelligent control system and application [M], mechanical industry press, 2015.
[12] L shiyong, Fuzzy control and intelligent control theory and application [M], Harbin Institute of Technology press, 2013. 